\title{
Computational Fluency and Strategy Choice Predict Individual and Cross-National Differences in Complex Arithmetic
}

ARTICLE in DEVELOPMENTAL PSYCHOLOGY · AUGUST 2015

Impact Factor: $3.21 \cdot$ DOI: $10.1037 / \operatorname{dev} 0000045 \cdot$ Source: PubMed

READS

19

3 AUTHORS, INCLUDING:

Elida V Laski

Boston College, USA

12 PUBLICATIONS 124 CITATIONS

SEE PROFILE 


\title{
Computational Fluency and Strategy Choice Predict Individual and Cross-National Differences in Complex Arithmetic
}

\author{
Marina Vasilyeva, Elida V. Laski, and Chen Shen \\ Boston College
}

\begin{abstract}
The present study tested the hypothesis that children's fluency with basic number facts and knowledge of computational strategies, derived from early arithmetic experience, predicts their performance on complex arithmetic problems. First-grade students from United States and Taiwan $(N=152$, mean age: 7.3 years) were presented with problems that differed in difficulty: single-, mixed-, and double-digit addition. Children's strategy use varied as a function of problem difficulty, consistent with Siegler's theory of strategy choice. The use of decomposition strategy interacted with computational fluency in predicting the accuracy of double-digit addition. Further, the frequency of decomposition and computational fluency fully mediated cross-national differences in accuracy on these complex arithmetic problems. The results indicate the importance of both fluency with basic number facts and the decomposition strategy for later arithmetic performance.
\end{abstract}

Keywords: cross-national, mathematics learning, arithmetic, fluency, strategy choice

A strong foundation in mathematics is critical for acquiring knowledge in scientific and technological disciplines (National Mathematics Advisory Panel, 2008). Within mathematics, mastery of arithmetic is one of the key early processes that provide building blocks for subsequent learning (Cowan et al., 2011; Jordan, Kaplan, Locuniak, \& Ramineni, 2007). A large body of research has examined the development of arithmetic skills in young elementary school students but much of this research has focused on single-digit problems (Barrouillet \& Lépine, 2005; Geary, BowThomas, Liu, \& Siegler, 1996; Imbo \& Vandierendonck, 2007; Lindberg, Linkersdörfer, Lehmann, Hasselhorn, \& Lonnemann, 2013). Investigating performance on more complex problems could provide insight into how early computational skills support further mathematics learning.

The idea that development in general, and mathematics learning in particular, involves integration of previous skills in service of the acquisition of more complex ones has been broadly discussed in the literature (e.g., Case, 1993; Fischer, 1980; Griffin, 2009). The present study examined this process in the context of arithmetic. First-grade students were presented with simple problems that had been taught in school (i.e., single-digit addition) and complex problems on which they had received little to no classroom instruction (i.e., mixed-digit and double-digit addition). We explored the extent to which children's knowledge of computa-

This article was published Online First August 24, 2015.

Marina Vasilyeva, Elida V. Laski, and Chen Shen, Lynch School of Education, Boston College.

Thanks are due to the schools and children in the United States and Taiwan who participated in this study and to Amy Hachigan, Anna Ermakova, and Marina Ivanova for their assistance with collecting and coding the data.

Correspondence concerning this article should be addressed to Marina Vasilyeva, Department of Counseling, Lynch School of Education, Boston College, Chestnut Hill, MA 02467. E-mail: mvasilyeva@bc.edu tional strategies and basic number facts, derived from early arithmetic experience, provides a means of solving complex problems. Further, we investigated whether strategy choice and basic number fact fluency might account not only for individual variability, but also cross-national differences, in performance on complex problems. Extant research has documented substantial differences between American and Asian elementary students in performance on single-digit problems (e.g., Geary et al., 1996). If performance on complex addition involves the integration of earlier skills, then one would expect cross-national differences on more complex problems as well, and that these differences could be mediated by knowledge of computational strategies and basic number facts.

\section{Arithmetic Strategies}

By first grade, children have been observed using different types of strategies to solve arithmetic problems. The three main categories of early arithmetic strategies are counting, decomposition, and retrieval (Geary, Fan, \& Bow-Thomas, 1992; Shrager \& Siegler, 1998). Counting involves enumerating either one or both of the addends (e.g., $6+3 . .7,8,9$ ). Decomposition involves transforming the original problem into two or more simpler problems (e.g., $6+5 \ldots 6+4=10,10+1=11$ ). Retrieval involves recalling a number fact stored in memory, rather than active computation. According to the overlapping waves model (Siegler, 1996), children make adaptive choices in their strategy use, switching from one strategy to another, depending on the type of problem presented. In this model, strategy choice is described in terms of a competition between accuracy and efficiency, whereby a child selects a strategy in order to maximize the likelihood of producing a correct response, while also attempting to increase the efficiency of problem solving.

An illustration of adaptive strategy choice can be seen in the study by Geary and colleagues (2004). Children were presented with single- and mixed-digit addition tasks (the latter included single- and double-digit addends). When solving mixed-digit prob- 
lems, first graders relied less on retrieval and more on counting, compared with single-digit problems. This increase in the use of counting strategies likely reflects an attempt to maximize the chances of getting the correct response: mixed-digit addition facts (e.g., $16+5$ ) are less likely to be stored in first graders' memory than single-digit facts, making a retrieval strategy less reliable. In terms of efficiency, even though counting is not as fast as retrieval, it can still be relatively quick in the case of single- and mixed-digit addition, especially if the child uses a count-on strategy, starting with the larger addend and adding by one the value of the smaller addend (e.g., $16+5 \ldots 17,18,19,20,21$ ). Thus, on mixed-digit problems, the use of a counting strategy increases the accuracy relative to retrieval, while still being fairly efficient.

Presenting children with more complex addition tasks, such as double-digit problems, as in the current study, poses a new set of challenges. It is highly unlikely that number facts, such as " $34+$ $28=62$," are stored in the child's memory, thereby there is less possibility of using retrieval, but at the same time, counting does not appear to be an attractive option either. Even the most efficient count-on strategies, which provide an acceptable option for mixeddigit problems, make the double-digit addition task extremely inefficient and error-prone. A more favorable alternative is offered by decomposition. For example, $34+28$ might be solved by separately adding the $10 \mathrm{~s}$ and the $1 \mathrm{~s}$, and then combining the results.

Can first graders solve double-digit addition problems using a decomposition strategy? Prior work (e.g., Fuson \& Kwon, 1992; Geary et al., 1996; Lindberg et al., 2013) has shown that first graders have several decomposition strategies in their repertoire, such as decomposition through doubles (e.g., $6+7=6+6+$ $1=12+1=13$ ) or through 10 (e.g., $6+7=6+4+3=10+$ $3=13$ ). Having a general idea of decomposition (i.e., breaking the target problem into several simplified problems) is useful for solving problems with double-digit addends, but may not be sufficient. On double-digit problems, decomposition often involves separating the $10 \mathrm{~s}$ and the $1 \mathrm{~s}$ (e.g., $24+13=[20+10]+[4+3]=37$ ). Knowledge of the base-10 number structure, thus, is critical for this type of decomposition. Children who are able to represent a number as a combination of $10 \mathrm{~s}$ and $1 \mathrm{~s}$ (e.g., 24 is two 10s and four 1s) should be more likely to apply this strategy, compared to children who tend to represent numbers as collections of single units. Indeed, a recent study found that kindergartners' ability to represent the base-10 structure of double-digit numbers predicted their use of a base-10 decomposition strategy in solving arithmetic problems (Laski, Ermakova, \& Vasilyeva, 2014).

Further, a successful execution of a decomposition strategy on double-digit problems appears to require a high degree of fluency with basic number facts. Decomposition strategies tend to use substantial working memory resources (DeStefano \& LeFevre, 2004; Geary, Hoard, Byrd-Craven, \& DeSoto, 2004; Imbo \& Vandierendonck, 2007), and having double-digit addends further increases the demand for these resources. Consider the problem $34+28$ : If the child adds the $10 \mathrm{~s}$ and then begins to add the $1 \mathrm{~s}$ using either counting or further decomposition, the sum of the tens may disappear from memory. If, however, the child is fluent with single-digit addition facts and can quickly retrieve them from memory, there is a better chance that final addition steps are completed successfully.

\section{Computational Fluency}

Computational fluency has been operationalized in different ways, yet the common thread among measures of fluency is that they capture the ability to solve simple arithmetic problems quickly and accurately (Carr \& Alexeev, 2011; Geary, Fan, \& Bow-Thomas, 1992). Computational fluency is attained through accumulated practice solving simple problems, which leads to memorizing basic number facts and the development of interconnected numeric knowledge (Baroody, 2006). It has been shown that both the amount of computational practice and student characteristics, in particular, working memory, account for individual differences in fluency (Bailey, Littlefield, \& Geary, 2012; Barrouillet \& Lépine, 2005).

Fluency has been implicated in performance on a range of mathematical tasks (e.g., Cowan et al., 2011; Whang \& Hancock, 1997). Investigators have shown that children's level of fluency affects the development of arithmetic strategies by facilitating the transition from the use of manipulatives to mental strategies (Carr \& Alexeev, 2011; Jordan, Hanich, \& Kaplan, 2003). There is a general agreement that computational fluency affects math performance by freeing working memory resources during problem solving. An ability to quickly retrieve number facts allows individuals to allocate more cognitive resources to other aspects of the problem, which is particularly important in the use of memoryintensive strategies, such as decomposition. Thus, fluency may interact with the use of decomposition on complex arithmetic problems by supporting the efficient execution of this strategy.

\section{Cross-National Differences in Arithmetic Performance and Instruction}

In cross-national investigations, Asian students tend to outperform their American peers in arithmetic and other mathematical tasks (Geary et al., 1996; Mullis, Martin, Foy, \& Arora, 2012; Stevenson, Chen, \& Lee, 1993). Differences exist not only in overall performance level but also in strategy use. For example, Geary and colleagues (1996) demonstrated cross-national differences in strategies used for single-digit addition. At the start of first grade, Chinese students relied mostly on decomposition and retrieval, whereas American students relied mostly on counting. At the end of first grade, the prevalent strategy among Chinese students became retrieval, whereas a large percentage of Americans continued to use counting. There were also cross-national differences in retrieval time. In fact, the frequency of correct retrieval and the mean retrieval time were the key predictors of children's accuracy on single-digit problems. Partialing out these variables eliminated the statistical difference in accuracy between Chinese and American participants. A parallel finding on singledigit addition was reported in a study where the analysis focused on students with a higher level of math skills (Geary et al., 1992).

The observed cross-national differences in children's arithmetic performance may reflect differences in math instruction. Two key differences are relevant to the present study. One is the extent to which instruction emphasizes practice with simple arithmetic problems. Classroom instruction in Asian countries-such as China, Japan, and Korea-involves intense computational practice in early grades, promoting a quick and accurate retrieval of basic number facts (e.g., Leung, 2001). This approach likely contributes 
to the high frequency with which retrieval is used by Asian students compared with their American peers.

Another notable aspect of early math instruction for which cross-national differences have been documented is the emphasis on decomposition strategy. Although first graders in the United States, as well as in Asian countries, are introduced to a variety of arithmetic strategies, including retrieval, counting and decomposition, there is a clear difference in the extent to which particular strategies are emphasized. Our examination of curricular materials in the countries participating in the present study revealed that in Taiwan instruction emphasized the use of decomposition, whereas in the United States there was no particular emphasis on one strategy over the other. Children in both countries are taught decomposition through doubles (e.g., $6+7=[6+6]+1=12+1$ ) and through 10 (e.g., $6+7=[6+4]+3=10+3)$, but there is more instruction on base-10 decomposition in Taiwan compared with that in the United States. Similarly, Fuson and Li (2009) reviewed textbooks widely used in China and the United States and found that Chinese textbooks promoted a base-10 decomposition method to a much greater extent than American textbooks. An analysis of math lessons in Chinese schools by Perry (2000) offered salient examples of first-grade teachers highlighting the base-10 decomposition strategy. This greater emphasis on base-10 decomposition may prove useful when children start solving complex arithmetic problems, such as multidigit addition, for which decomposition is a particularly effective strategy.

No cross-national study to date has compared young students' performance on complex arithmetic problems. These problems may show a different pattern of strategy use compared with singledigit problems. As retrieval becomes unavailable, children are likely to rely on "backup" strategies, such as decomposition or counting. On the basis of the tendencies shown by Asian and American students in earlier studies with simple addition (Geary et al., 1996) and the differences in arithmetic instruction, these students can be expected to vary in their choice of strategies when solving more complex problems. The extent of such differences in strategy use and their role in understanding cross-national differences in accuracy on complex arithmetic problems are the focus of the present study.

\section{Present Study}

Our investigation pursued three interrelated goals. First, we aimed to explore the strategies first graders used to solve complex arithmetic problems and compare them with the strategies used on simple problems. To address this goal, children were given a task that included three types of items - single-digit (SD), mixed-digit (MD), and double-digit (DD) addition. Following the overlapping waves model (Siegler, 1996), we expected that strategy choice would vary with problem difficulty. Specifically, we predicted that the use of decomposition would increase on DD, compared with $\mathrm{SD}$ and MD problems; in contrast, the use of counting strategies would decrease on DD problems as counting becomes increasingly inefficient.

Our second goal was to compare the use of strategies by American and Taiwanese students. On the basis of evidence from prior work, we expected that in solving SD addition problems, Taiwanese students would use retrieval more frequently than their American counterparts. Further, on the basis of our analysis of instruc- tional practices in the two countries, we hypothesized that when solving MD and DD problems, Taiwanese students would use decomposition more frequently than American students. The American students, in turn, would rely more than their Taiwanese counterparts on counting strategies as a backup for retrieval. Thus, even if the general tendency would be to increase the use of decomposition from SD to DD problems, this increase would be more pronounced in Taiwanese students.

Our third set of questions addressed the relation between first graders' strategies and accuracy on double-digit addition problems. Specifically, we examined whether strategy choice accounted for differences between American and Taiwanese participants' performance on these problems. We expected to see differences in accuracy between the two groups because of prior findings showing that Taiwanese students outperform their American peers in a wide range of mathematical tasks (e.g., Mullis, Martin, Foy, \& Arora, 2012). On the basis of the analysis of the double-digit addition task, we hypothesized that higher levels of accuracy on this task are associated with a more frequent use of decomposition strategy and greater computational fluency. Further, we hypothesized that accurate execution of the decomposition strategy may depend on fluency with basic computational facts. Thus, we tested the hypothesis that the use of the decomposition strategy and computational fluency both mediate cross-national differences in accuracy on double-digit addition and interact with each other in predicting accuracy.

\section{Method}

\section{Participants}

The study included 152 first graders: 92 from Massachusetts, United States, and 60 from Taipei, Taiwan. The samples were comparable in age and gender distribution (United States: $M$ age 7.2 years, $52 \%$ female; Taiwan: $M$ age 7.4 years, $53 \%$ female). In cross-national investigations, it is difficult to match participants' socioeconomic backgrounds because countries vary in income criteria used to define socioeconomic status (SES). Thus, in the present study, we used parental education criteria to ensure the comparability of the samples. In the United States, children were from suburbs largely populated by highly educated professionals $(75 \%$ of adults with Bachelor's or higher degree). In Taiwan, where demographic data were not publicly available, we relied on information about schools' reputation provided by local researchers. On the basis of this information, students were from schools serving high educational and professional status families.

On the basis of a careful examination of math curriculum used in participating schools (United States: Investigations in Number, Data and Space, TERC, 2008 and Everyday Mathematics, The University of Chicago School Mathematics Project, 2012; Taiwan: The Nine-Year School Curriculum/First-Grade Elementary School, Ministry of Education of Taiwan, 2011) and interviews with teachers who taught our study participants, we determined that the children from both countries had a substantial amount of practice (starting in kindergarten) with SD addition and some practice (in the first grade) with MD addition but had not received classroom instruction on DD addition by the time of the study. It is possible, of course, that some children were exposed to such 
problems outside of school, but still this exposure would be relatively minimal, compared to SD addition.

\section{Design Overview}

First graders met individually with an experimenter for two sessions. In the first session, children completed a number representation task. The purpose of administering this task was to assess children's understanding of the base-10 number structure in order to establish that they had the knowledge necessary to execute a decomposition strategy on multidigit addition problems. In the second session, which was administered about two weeks later, children completed the arithmetic task. All testing was conducted at the end of academic year.

\section{Materials and Procedure}

Number representation task. Materials for this task included two kinds of plastic blocks: 100 small cubes (unit-blocks), each representing a single unit, and 20 longer bars (10-blocks), with 10 single-unit segments marked. Both types of blocks were made from the same material and were the same color. In addition to blocks, materials included cards displaying the written numerals that were to be represented.

The procedure was based on the block-task used in earlier studies as a measure of the base-10 knowledge (Miura, 1987; Saxton \& Towse, 1998). The child was presented with two traysone containing single unit-blocks and the other containing 10blocks. The tester explained that the blocks could be used to show numbers and introduced the task by showing a card with a numeral and then demonstrating the appropriate number of blocks to represent that numeral. Each child was given five test trials with numbers $12,16,28,34$, and 61 presented in a random order. The child was asked to show the number displayed on the card using blocks. The tester recorded how many unit- and 10-blocks the child used to represent the target number. Responses were coded into one of four categories, as presented in Table 1.

Arithmetic task. Materials included 24 addition problems, each printed on a separate sheet of paper. The items were divided into three blocks, always presented in the same order. The first and the last blocks each included six SD problems, whereas the middle block included 12 more complex problems (eight MD and four DD). This order of blocks was intended to encourage children by presenting simpler problems at the beginning of testing and to provide relief toward the end of testing. Within each block, items were presented in random order. Half of the SD problems had sums up to 10 (e.g., $6+2$ ) and the other half had sums over 10 (e.g., $5+7$ ). Similarly, half of the MD and DD problems did not involve carry-over (e.g., $15+3 ; 11+17$ ) and the other half involved carry-over (e.g., $26+8 ; 25+37$ ).

A smaller number of DD problems were included due to our observation during pilot testing that some first graders got frustrated by having to solve a large number of challenging problems that they never did in school and this affected their performance on subsequent trials. Thus, we sought a balance between including a sufficient number of items to yield a reliable measure of performance and not overwhelming children with difficult problems. To examine the reliability of our assessment instrument, we computed the Kuder-Richardson statistics (KR-20) for each type of item. KR-20 reflects the internal consistency of the scale; it is a version of Cronbach's alpha computed for dichotomous responses (correct/incorrect). KR-20 values above 0.70 are considered good indicators of reliability for test instruments with fewer than 50 items (Rosenthal \& Rosnow, 1991; Salkind, 2010). Our analysis indicated that the KR-20 values were comparable across the problem types and exceeded the 0.70 criterion in all three cases $(0.79$, 0.75 , and 0.76 for SD, MD, and DD problems, respectively).

The procedure involved presenting children with addition problems, and asking them to solve the problem and then to explain how they arrived at the solution. Each problem was presented in written form and accompanied by the tester's question, for example, "How much is $5+7$ ?" Strategy explanations were prompted by the question "How did you figure it out?" which was posed right after the child provided a response to the problem. Participants were presented with one problem at a time, allowing as much time as needed to solve the problem. They were not provided with any supplies, such as pencil and paper, but were allowed to use fingers or count out loud if they wished. Children's responses were audio-recorded. The recordings were later used to measure response times and identify strategies on each trial.

Strategy coding. Children's responses were coded following the strategy assessment scheme presented by Geary and colleagues (Geary, Bow-Thomas, Liu, \& Siegler, 1996; Geary, Hoard, ByrdCraven, \& DeSoto, 2004). The data used in the coding process included children's explanations and response times, as well as the tester's notes made while the child was solving the problem and explaining the solution. Specifically, the tester observed the child during the assessment and recorded any overt signs of strategy use (e.g., if the child counted out loud or held up fingers, the tester would mark down a counting strategy). When there were no overt behaviors, the tester asked the participant how he or she "figured it out" after an answer was provided. After the assessment, audiorecordings were transcribed to document children's explanations. Response times were recorded as the interval between the point when the tester presented the problem and the start of the child's

Table 1

Categories of Number Representations

\begin{tabular}{|c|c|c|}
\hline Category & Description & Examples \\
\hline Canonical base-10 & $\begin{array}{l}\text { Using the largest possible number of ten-blocks to } \\
\text { represent tens and unit-blocks to represent ones }\end{array}$ & $34=3$ 10-blocks +4 unit-blocks \\
\hline Non-canonical base-10 & Using some ten-blocks and more than 9 unit-blocks & $34=210$-blocks +14 unit-blocks \\
\hline Single unit collection & Using only single-unit blocks & $34=34$ unit-blocks \\
\hline Other & Guessing, no-response, or lack of task understanding & $34=2010$-blocks blocks +14 unit-blocks (unit confusion) \\
\hline
\end{tabular}


response. This kind of combination of behavioral observations during problem solving, retrospective self-reports, and latency criteria has been found to lead to valid strategy classifications (Rittle-Johnson \& Siegler, 1999; Siegler, 1987).

Children's strategies were coded as one of three types-counting, decomposition, and retrieval. Counting strategies involved enumerating each unit for one or both addends. Decomposition involved breaking the problem into simpler problems. This could be done either using a previously memorized number fact or the base-10 property of the number system (e.g., when adding doubledigit numbers, children add the $1 \mathrm{~s}$, then the $10 \mathrm{~s}$, and finally combine the two results). Retrieval referred to instances when children provided a quick response with no overt evidence of counting or decomposition and reported knowing the answer. As a criterion of quick retrieval we used a 3-s limit on response time. This criterion is based on prior studies in which a similar time limit was imposed on children's responses to prevent them from carrying out calculations using counting or decomposition strategies (Booth \& Siegler, 2008; Jordan, Hanich, \& Kaplan, 2003). When the child's explanation did not fit into one of the three main categories, it was coded as "other." The "other" category included instances when the child reported guessing or could not articulate a strategy and if the strategy was not clear from either overt behaviors or verbal explanations. The same criteria applied to coding strategies on $\mathrm{SD}, \mathrm{MD}$, and $\mathrm{DD}$ problems.

To calculate interrater reliability for the coding of strategies, $25 \%$ of all responses were coded independently by two raters. The level of agreement between them was $96 \%$. Later, the raters discussed with each other the few cases where they initially disagreed and together arrived at the final code.

Fluency. As an indicator of fluency with basic number facts, we used a measure that combined accuracy and response time. We used both parameters because measuring fluency by response time only can be problematic, particularly with children. Some children produce incorrect responses quickly, but do so by guessing or systematically using an incorrect strategy. As a result, children who are truly fluent may be combined with children who have not mastered basic number facts and lack metacognitive awareness of the limitations of their knowledge. Thus, we operationalized computational fluency as the ability to retrieve basic addition facts from memory quickly and accurately. Using audiotaped records of testing sessions, we identified SD trials on which children (a) used quick retrieval (i.e., showed no behavioral indications of using other strategies, reported knowing the answer, and responded within three seconds) and (b) produced a correct response. The proportion of such trials out of all SD trials served as a measure of computational fluency.

\section{Results}

First, we present the analysis of children's performance on the number representation task, which was used to establish whether children had the prerequisite knowledge to execute the decomposition strategy on double-digit problems. Next, we examine performance on the arithmetic task, presenting results in the order of our research questions. In examining arithmetic skills, we analyzed both accuracy and strategies as a function of problem type (SD, $\mathrm{MD}, \mathrm{DD})$ and compared the patterns across countries. To anticipate, we found the largest differences between the two countries in children's performance on DD addition. Thus, in the next set of analyses, we focused specifically on DD problems investigating whether the use of decomposition strategy and computational fluency mediated cross-national differences in DD addition accuracy.

\section{Number Representation Task}

The accuracy of performance on this task was coded as follows: a response was considered correct if the value of the blocks used by a child added up to exactly the number shown on the card. For example, if " 28 " was represented with 28 single units or two 10-blocks and eight single units or one 10-block and 18 single units, it was coded as correct. The results showed a high level of accuracy of constructed representations (98\%) in both countries.

Next, we examined the frequency with which children produced base-10 representations of DD numbers. We found that such representations were created on most trials in both countries (United States: 91\%, Taiwan: 97\%). Almost all of the base-10 representations (United States: 99\%, Taiwan: 100\%) were canonical, whereby all the 10 s were represented with 10-blocks, as opposed to a mix of unit- and 10-blocks. To statistically compare the frequency of base-10 representations across countries, we conducted a Mann-Whitney test (which was used instead of a $t$ test because both samples had highly skewed distributions). The results showed no significant differences between the two countries $(p=$ .23). Thus, American and Taiwanese students demonstrated a comparably high level of knowledge of the base-10 structure of DD numbers, which is a key prerequisite for the use of base-10 decomposition strategies in DD addition.

Looking at the performance of individual participants, we found that the majority of children from both countries $(95 \%$ in the United States and 97\% in Taiwan) constructed base-10 representations on at least four out of five trials. The children who failed to produce at least four base-10 representations in this task (five from the United States and two from Taiwan) were not included in subsequent analysis.

\section{Arithmetic Task: Examining Performance as a Function of Problem Type and Country}

Prior to the main analysis of performance on the arithmetic task, we conducted a preliminary analysis to examine effects of demographic factors - gender and age- on accuracy and strategy use. The analysis involved a multivariate analysis of variance (MANOVA) with five outcome variables: overall accuracy (percentage correct) and the proportion of trials on which children used each of the four strategy categories (retrieval, counting, decomposition, other). The MANOVA showed that, for all these outcomes, gender and age did not produce significant main effects or interactions (all $p \mathrm{~s}>.05$ ) and thus they were eliminated from further consideration. Also prior to the main analysis, we transformed participants' scores on all of the proportional variables using the arcsine transformation to improve normality of data (Cohen, Cohen, Vest, \& Aiken, 2014).

Accuracy and response time. To examine accuracy on the addition task, we calculated percentage of correct responses in solving each of the three problem types (see Figure 1). A 3 (Problem Type) $\times 2$ (Country) analysis of variance (ANOVA) 


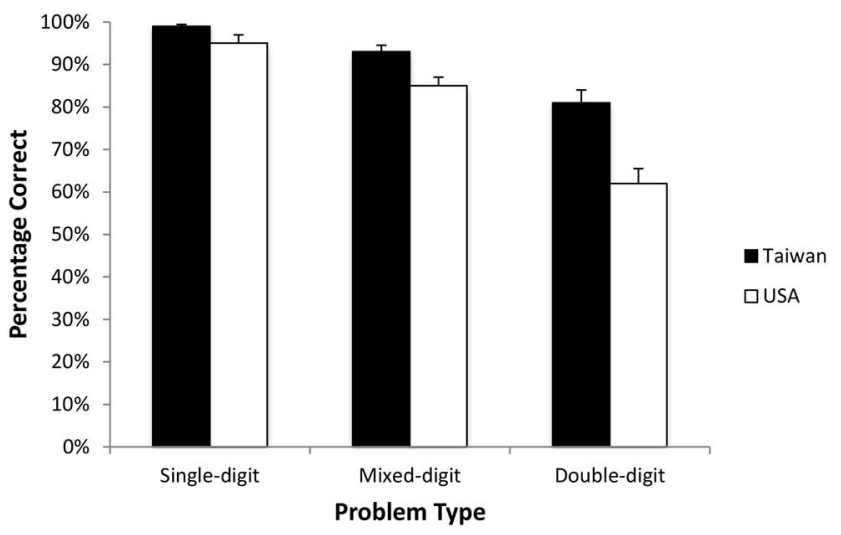

Figure 1. Accuracy of responses on three types of addition problems.

with accuracy as the dependent variable revealed a main effect of country, $F(1,150)=30.90, p<.001, \eta_{\mathrm{p}}^{2}=.17$, indicating an overall advantage of Taiwanese over American students. The effect of problem type was also significant, $F(2,300)=70.32, p<$ $.001, \eta_{\mathrm{p}}^{2}=.32$; least significant difference (LSD) pairwise comparisons showed that the accuracy on SD problems was higher than on MD problems, which in turn was higher than on DD problems, all $p s<.05$.

In addition to main effects, there was an interaction between country and problem type, $F(2,300)=10.38, p<.01, \eta_{\mathrm{p}}^{2}=.07$. To better understand the interaction, tests of simple effects were conducted. They showed no country differences for SD problems, $F(1,150)=1.92, p=.17, \eta_{\mathrm{p}}^{2}=.01$, but an effect of country on both MD problems, $F(1,150)=5.98, p=.02, \eta_{\mathrm{p}}^{2}=.06$, and DD problems, $F(1,150)=33.27, p<.001, \eta_{\mathrm{p}}^{2}=.20$. As illustrated in Figure 1, the effect size was substantially larger for DD problems than for MD ones. Thus, the advantage of Taiwanese over American students progressively increased as a function of problem complexity.

Next, we examined response times on each of the three problem types, which are shown in Figure 2. A 3 (Problem Type) $\times 2$ (Country) ANOVA with response time as the dependent variable revealed main effects of country, $F(1,150)=36.12, p<.001$, $\eta_{\mathrm{p}}^{2}=.23$, and problem type, $F(2,300)=142.48, p<.001, \eta_{\mathrm{p}}^{2}=$ .54. Pairwise LSD comparisons showed that the response time on SD problems was lower than on MD problems, which in turn was lower than on DD problems, all $p s<.05$. As in the analysis of accuracy, we found an interaction between country and problem type, $F(2,300)=19.99, p<.001, \eta_{\mathrm{p}}^{2}=.14$. Tests of simple effects showed that there were significant country differences on all three types of problems, yet the effect size on DD addition was substantially larger than the effect sizes on the other two problem types: SD problems, $F(1,150)=9.94, p<.01, \eta_{\mathrm{p}}^{2}=.07$; MD problems, $F(1,150)=11.13, p<.01, \eta_{\mathrm{p}}^{2}=.08$; DD problems, $F(1,150)=38.48, p<.001, \eta_{\mathrm{p}}^{2}=.24$. Thus, response time findings largely mirrored accuracy results. One exception was the performance on SD problems, where we found no country differences in accuracy, but significant differences in response times favoring Taiwanese students. Other than that, the general tendency held: Taiwanese students were both more accurate and faster in producing responses and these differences were most salient on DD addition.
Strategy use. The proportions of SD, MD, and DD trials on which children used a particular type of strategy are presented in Table 2. As shown in the table, retrieval was the most frequent strategy on SD addition problems in both counties, although its use was higher among Taiwanese first graders. It is interesting to note that a small percentage of responses on MD and DD problems were also coded as retrieval, and most of them were produced by Taiwanese students. It is not entirely clear whether these students indeed retrieved complex number facts, such as $25+37=62$, from memory or instead carried out mental computations very quickly. American students did not produce any responses on DD problems that could be coded as retrieval; at the same time, $14 \%$ of their responses on this problem type (a larger percentage, compared to Taiwan or other problem types in the United States) were categorized as other. A closer examination of the data revealed that it was in part due to American students' greater tendency, compared to Taiwanese counterparts, to refuse solving problems that had not been previously taught.

Our statistical analyses focused on the use of the other two strategies, counting and decomposition, that together accounted for the majority (over $80 \%$ ) of responses on MD and DD addition problems. A 3(Problem Type) $\times 2$ (Country) ANOVA using the proportion of counting as the dependent variable showed a main effect of country, $F(1,150)=26.11, p<.001$, $\eta_{\mathrm{p}}^{2}=.15$, with American students using counting more frequently than the Taiwanese $(M=.40, S D=.29$ and $M=.15, S D=.20$, respectively). The effect of problem type was also significant, $F(2,300)=15.22$, $p<.001, \eta_{\mathrm{p}}^{2}=.09 ;$ LSD analyses showed an increase in the use of this strategy from SD to MD problems $(M=.24, S D=.22$ and $M=.35, S D=.31$, respectively), a decrease from MD to DD problems $(M=.35, S D=.31$ and $M=.23 S D=.26$, respectively), and no differences between SD and DD problems. The interaction between problem type and country was not significant. Thus, even though the extent to which children relied on counting varied substantially between United States and Taiwan, the pattern of use of this strategy as a function of problem type was parallel in the two countries, as shown in Figure 3A.

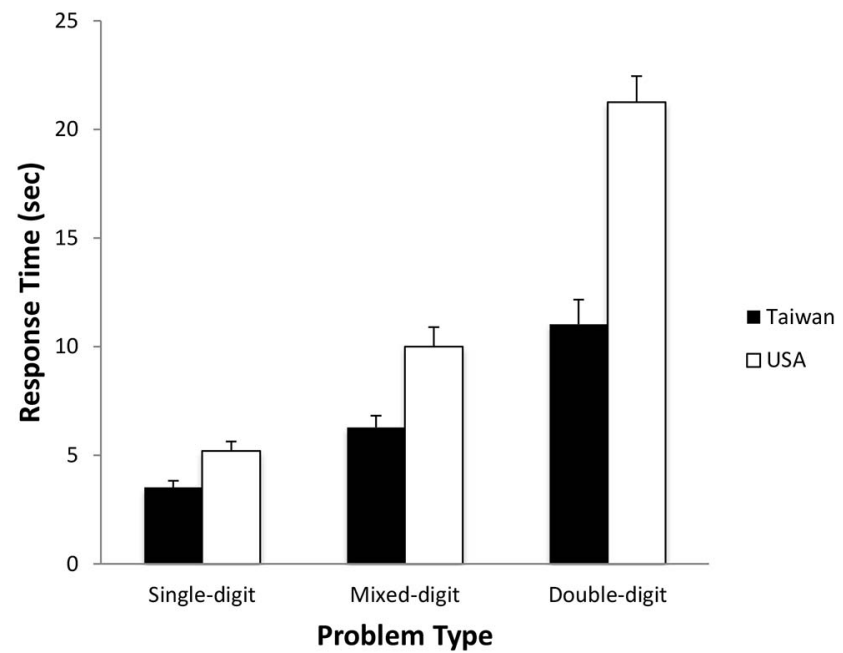

Figure 2. Response times on three types of addition problems. Panel A: Counting strategy. Panel B: Decomposition strategy. 
Table 2

Proportion of Strategies Used on Three Types of Addition Problems

\begin{tabular}{lccc}
\hline \multicolumn{1}{c}{ Strategy } & $\begin{array}{c}\text { Single-digit } \\
\text { addition problems }\end{array}$ & $\begin{array}{c}\text { Mixed-digit } \\
\text { addition problems }\end{array}$ & $\begin{array}{c}\text { Double-digit } \\
\text { addition problems }\end{array}$ \\
\hline $\begin{array}{l}\text { United States } \\
\text { Counting }\end{array}$ & $.32(.03)$ & & \\
Decomposition & $.20(.02)$ & $.49(.04)$ & $.38(.04)$ \\
Retrieval & $.46(.03)$ & $.42(.04)$ & $.48(.05)$ \\
Other & $.02(.005)$ & $.02(.01)$ & $.00(.00)$ \\
Taiwan & $.13(.03)$ & $.08(.02)$ & $.14(.03)$ \\
Counting & $.23(.03)$ & $.22(.04)$ & $.10(.03)$ \\
Decomposition & $.63(.04)$ & $.63(.05)$ & $.75(.05)$ \\
Retrieval & $.01(.004)$ & $.09(.02)$ & $.07(.03)$ \\
Other & $.06(.02)$ & $.08(.02)$ \\
\hline
\end{tabular}

Note. Standard errors are indicated in parentheses.

With respect to decomposition, a 3(Problem Type) $\times 2$ (Country) ANOVA revealed a main effect of country, $F(1,150)=$ 11.56.11, $p<.01, \eta_{\mathrm{p}}^{2}=.05$, with Taiwanese students using decomposition more frequently than American students $(M=.54$, $S D=.27$ and $M=.37, S D=.25$, respectively). The main effect of problem type was also significant, $F(2,300)=108.87, p<$ $.001, \eta_{\mathrm{p}}^{2}=.42$, with follow-up LSD analyses showing that all pairwise comparisons among $\mathrm{SD}, \mathrm{MD}$, and $\mathrm{DD}$ problems were significant $(M=.21, S D=.20 ; M=.52, S D=.34$; and $M=.61$, $S D=.45$, respectively). Furthermore, there was an interaction between problem type and country, $F(2,300)=10.04, p<.001$, $\eta_{\mathrm{p}}^{2}=.06$, which is illustrated in Figure 3B. Simple effects tests showed that the use of decomposition by Taiwanese students significantly increased between SD and MD problems, and then again between MD and DD problems $(M=.23, S D=.21 ; M=$ $.63, S D=.40$; and $M=.75, S D=.40$, respectively). In contrast, the use of this strategy by American students significantly increased between SD and MD but not between MD and DD problems $(M=.20, S D=.20 ; M=.42, S D=.38$; and $M=.48$, $S D=.45$, respectively).

The analyses of strategy use across problem types indicated a clear tendency to decrease the use of counting and increase the use of decomposition when solving DD problems, as opposed to simpler problem types. Our task analysis predicted this pattern because it suggested that decomposition offers a more efficient way to add double-digit numbers than counting. To examine the issue of strategy efficiency, we calculated the response times when children used decomposition and counting on DD problems. Consistent with our task analysis and the idea that children use decomposition more frequently on DD problems because it is more efficient on those problems than counting, we found that children solved DD problems substantially faster when they used decomposition than when they used counting. This pattern was true for both countries: Taiwan ( $M$ response time: $24.8 \mathrm{~s}$ vs. $9.7 \mathrm{~s}$ for counting and decomposition, respectively) and United States (34.7 s vs. $18.8 \mathrm{~s}$ for counting and decomposition, respectively).

Fluency. The measure of computational fluency was computed based on SD trials as described in the Method section. We found that the first graders from the United States and Taiwan differed significantly on this measure, $t(150)=3.56, p<.001$, with the Taiwanese students showing higher fluency. It is interesting to note that for the Taiwanese sample, the average computational fluency score was very close to the frequency of retrieval $(M=0.62, S D=.28$ and $M=0.63, S D=.28$, respectively $)$ whereas in the U.S. sample the computational fluency score was somewhat lower than the frequency of using retrieval $(M=0.40$, $S D=.27$ and $M=0.46, S D=.27$, respectively). In other words, when the Taiwanese students used a retrieval strategy, they were almost always accurate, whereas the American students using retrieval were sometimes inaccurate.
A. Counting Strategy

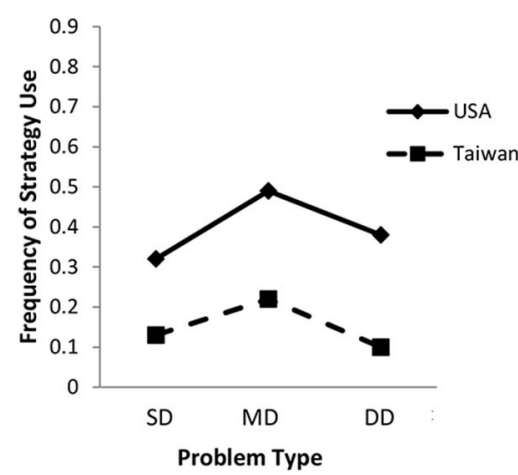

B. Decomposition Strategy

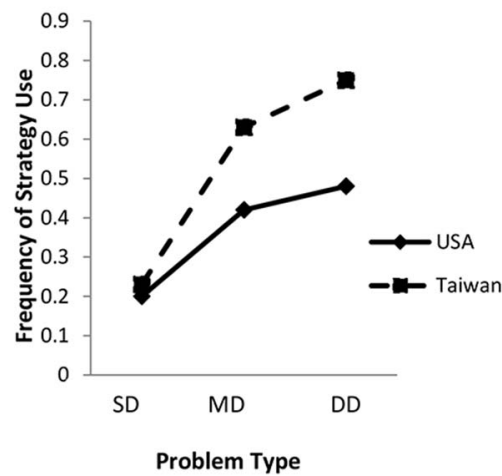

Figure 3. Patterns of strategy use across three types of addition problems. 


\section{Mediation Analysis of Cross-National Differences on DD Addition}

Preliminary analysis. Prior to conducting the mediation analysis, we computed correlations, examining how the accuracy of DD addition was related to computational fluency and to the frequency of various strategies used on DD problems. Because the relations between variables might differ in the two countries, the correlations were calculated for each country separately. Table 3 shows that the pattern of results obtained with American students was largely parallel to that obtained with Taiwanese students (with the exception of "other" strategy use, which was negatively correlated with accuracy in the United States and was not related to accuracy in Taiwan). In both countries, children's accuracy on DD addition was positively correlated with fluency and frequency of decomposition, but negatively correlated with the use of counting. We conducted the Fisher's $z$ test to compare the strength of the associations between corresponding variables in the two countries. It showed that correlation coefficients did not differ significantly across countries (accuracy and fluency: $z=.59$, accuracy and decomposition strategy: $z=.97$, accuracy and counting strategy, $z=-.84)$.

Strategy for estimation of mediation models. Whereas the correlational analysis indicates that both strategy choice and fluency are associated with individual differences in accuracy within each country, our key question concerned a potential role of these factors in relation to the observed cross-national differences in accuracy. As reported earlier, there were significant differences between the two countries on the variables of interest, with Taiwanese students showing greater fluency and more frequent use of decomposition strategy and higher accuracy on DD addition. We hypothesized that the cross-national differences in fluency and decomposition strategy use accounted, partially or fully, for the corresponding differences in accuracy.

To test this hypothesis, we conducted a mediation analysis, examining indirect effects of country on DD addition accuracy through two parallel paths-via computational fluency and via frequency of decomposition. Further, we considered the possibility that these potential mediators interact with each other. For exam- ple, the accurate execution of the decomposition strategy may depend on the level of fluency with basic number facts so that higher fluency facilitates the positive relation between the use of decomposition and DD addition accuracy. Conversely, the positive relation between fluency and DD accuracy may be strengthened by the use of decomposition because computational fluency is particularly critical for this strategy, compared to other strategies, such as counting. To test this possibility, we included an interaction term (Decomposition Strategy $\times$ Fluency) in the model, which allowed us to examine a moderated mediation effect for both mediators.

Testing for indirect effects involved estimating the regression coefficients for a number of pathways as outlined by Hayes (2013): (1) path $a_{1}$ : country to computational fluency; (2) path $b_{1}$ : computational fluency to accuracy; (3) path $a_{2}$ : country to frequency of decomposition; (4) path $b_{2}$ : frequency of decomposition to accuracy; (5) path $c$ : country to accuracy; (6) path $c^{\prime}$ : country to accuracy, controlling for fluency and strategy. To explicitly test for mediation, we used bias-corrected bootstrapping to estimate confidence intervals for the indirect effects (Hayes, Preacher, \& Myers, 2011; MacKinnon, 2008; Preacher \& Hayes, 2008). The SPSS macro called PROCESS, created by Preacher and Hayes (2004), was used to randomly select 10,000 samples with replacement from the complete data file. Regression coefficients were estimated for each of the bootstrap samples and averaged across all samples. The model we tested included two parallel mediators that simultaneously served as each other's moderator. The algorithm for estimating indirect effects within this model was created with the assistance of Professor Hayes through personal communication (2015). By adding the interaction between the two mediators to the analysis we were able (a) to model fluency as moderating the indirect effect through frequency of decomposition and (b) to model frequency of decomposition as moderating the indirect effect through fluency.

Moderated mediation findings. Figure 4 presents the results of the mediation analysis. The model depicted in this figure accounted for $39 \%$ of variance in DD accuracy. The indirect effect of country on DD accuracy through computational fluency $\left(a_{1} \times b_{1}\right)$ was estimated as $0.04,95 \%$ CI: [0.02; 0.08]; the indirect effect

Table 3

Correlations Between Accuracy, Fluency, and Strategy

\begin{tabular}{|c|c|c|c|c|c|}
\hline & $\begin{array}{c}\text { DD } \\
\text { accuracy }\end{array}$ & $\begin{array}{l}\text { Computation } \\
\text { fluency }\end{array}$ & $\begin{array}{c}\text { DD } \\
\text { decomposition }\end{array}$ & $\begin{array}{c}\text { DD } \\
\text { counting }\end{array}$ & $\begin{array}{l}\text { DD } \\
\text { other }\end{array}$ \\
\hline \multicolumn{6}{|l|}{ United States } \\
\hline DD accuracy & 1 & & & & \\
\hline Computation fluency & $.35^{* * *}$ & 1 & & & \\
\hline DD decomposition & $.52^{* * *}$ & .20 & 1 & & \\
\hline DD counting & $-.23^{*}$ & $-.34^{* *}$ & $-.78^{* *}$ & 1 & \\
\hline DD other & $-.27^{*}$ & -.06 & $-.45^{* *}$ & -.18 & 1 \\
\hline \multicolumn{6}{|l|}{ Taiwan } \\
\hline DD accuracy & 1 & & & & \\
\hline Computational fluency & $.26^{*}$ & 1 & & & \\
\hline DD decomposition & $.39^{*}$ & .16 & 1 & & \\
\hline DD counting & $-.36^{* *}$ & $-.34^{* * *}$ & $-.57^{* *}$ & 1 & \\
\hline DD other & -.04 & -.17 & $-.74^{* *}$ & -.14 & 1 \\
\hline
\end{tabular}




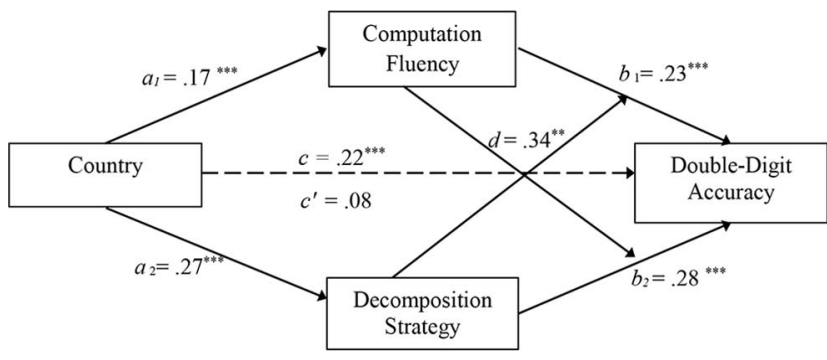

Figure 4. Computational fluency and decomposition strategy as mediators of cross-national differences in the accuracy of double-digit (DD) addition. ${ }^{* * * *} p<.001$.

through decomposition strategy $\left(a_{2} \times b_{2}\right)$ was estimated as 0.08 , 95\% CI: [0.04; 0.13]. Neither confidence interval contained the value of 0 , indicating that both indirect effects were statistically significant. As shown in Figure 4, the frequency of decomposition strategy and computational fluency fully mediated cross-national differences, as the relation between country and the DD accuracy became nonsignificant after the mediators were included in the model.

In addition to the main effects of the two mediators, the interaction term was also significant $(p=.02)$, indicating that the indirect effect of fluency was moderated by the frequency of decomposition and vice versa. The nature of the interaction is illustrated in Figure 5, which displays accuracy estimates for students with varying levels of computational fluency and decomposition strategy use (one standard deviation above and below the mean). The figure shows that both high fluency and a frequent use of decomposition strategy are associated with better accuracy. At the same time, the students who frequently use decomposition demonstrate a greater difference in accuracy as a function of fluency, compared to the students who are low on the use of decomposition. Thus, high fluency provides an additional boost for the accuracy of performance in children who frequently use decomposition.

\section{Discussion}

Recognizing the importance of arithmetic skills in mathematical learning, investigators have produced a large body of research examining the development of these skills in elementary school students (e.g., Geary et al., 1996; Göbel, Watson, Lervåg, \& Hulme, 2014; Jordan et al., 2007; Lindberg et al., 2013). The present study contributed to this area of research in several ways. First, we extended the types of problems previously studied by presenting first graders with double-digit addition tasks. This approach allowed us to systematically explore strategy choice as a function of problem complexity across a wider range of problems than in earlier studies. Second, we examined the joint contribution of computational fluency and decomposition strategy, as well as the interaction between these two factors, in predicting performance on double-digit addition. We established that both fluency and strategy choice serve as mediators of cross-national differences in performance on complex arithmetic tasks, suggesting a possibility of a causal pathway. In future research, a longitudinal or experimental design can be used to examine causal relations between these skills as they emerge.
Together, our findings indicate that first graders use their knowledge of number facts and arithmetic strategies to solve more complex less familiar problems. These results add to our understanding of mathematical skill development by identifying specific basic-level skills involved in complex arithmetic and demonstrating that differences in these skills contribute to individual and group-level variability in children's performance on advanced problems. Although the hierarchical relation between lower and higher level mathematical skills has been a long-standing theoretical premise (Fischer, 1980; Griffin, 2009), our results provide clear empirical evidence of this relation within a particular strand of mathematics.

\section{Strategy Choice as a Function of Problem Complexity}

It has been argued that strategy choice is largely determined by considerations of efficiency and accuracy: a selected strategy often reflects the most efficient among available options that is likely to lead to an accurate response (Siegler, 1987). Problem complexity affects both the efficiency and accuracy of strategy execution and thus can be expected to play a key role in choosing a strategy. Indeed, prior work has provided evidence for the role of problem complexity in strategy choice (e.g., Geary et al., 2004; Imbo \& Vandierendonck, 2007; Shrager \& Siegler, 1998). In the present study, extending the range of problem complexity allowed us to further test the interplay between efficiency and accuracy in strategy selection. We hypothesized that counting strategies, which present an acceptable alternative to retrieval on SD and to some degree MD problems, would be extremely inefficient for DD addition. This may push children to decrease the use of counting in favor of decomposition on more complex problems. In the following paragraphs, we discuss our results revealing precisely this pattern of strategy shift as a function of complexity.

Counting. Our study showed that the use of counting increased significantly from single- to mixed-digit problems, which is consistent with theoretical predictions based on the competition between accuracy and efficiency and replicates prior results (Geary et al., 2004). Further, the present study produced a unique finding: a decrease in the use of counting from mixed- to doubledigit problems. This pattern-an increase in the frequency of counting as a function of problem complexity, followed by a

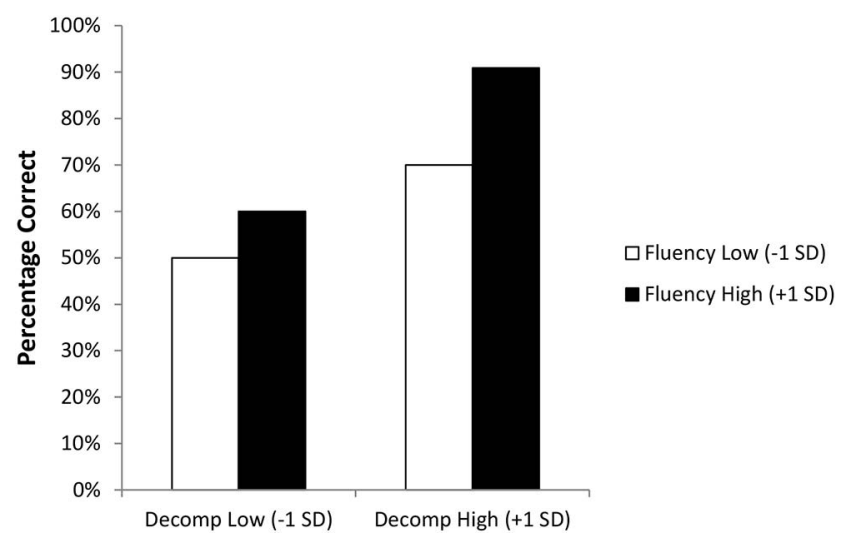

Figure 5. Interaction between strategy and fluency in predicting accuracy on double-digit (DD) problems 
decrease as a function of further complexity-was seen in both Taiwanese and American children, despite the fact that the overall levels of using this strategy were different (lower for Taiwanese). The observed pattern likely reflects children's sensitivity to a decreased efficiency of counting in the case of double-digit problems, which is clearly reflected in their response times.

Decomposition. The use of decomposition strategy as a function of problem complexity followed a different pattern than the counting strategy. Namely, there was a general increase in the frequency of decomposition with the increase in problem complexity. Taiwanese students showed a steady increase in the frequency of decomposition from SD to MD and to DD problems. American students demonstrated a substantial increase between SD and MD problems, but a small (and only marginally significant) increase from MD to DD problems. As a result, Taiwanese participants showed an overwhelming preference for decomposition over counting on DD addition ( $75 \%$ and $10 \%$, respectively), whereas American students' preference for decomposition over counting was smaller, albeit significant (48\% and $38 \%$, respectively).

We considered the possibility that the differential use of decomposition by our participants may have reflected differences in their understanding of the base-10 number structure. That is, if children tend to think of DD numbers as collections of single units, as opposed to combinations of tens and ones, they may be less likely to use decomposition, which involves breaking numbers into tens and ones, as a computational strategy. Thus, we examined our participants' ability to represent the base-10 structure of DD numbers using a block task, in which children had to show two-digit numbers using blocks that included cubes representing single units and bars representing 10 units. This task was developed by Miura and colleagues (Miura \& Okamoto, 1989; Miura, Okamoto, Kim, Steere, \& Fayol, 1993) who used it to document differences between American and East Asian students. In these studies, Asian first graders tended to produce base-10 representations (combinations of 10-bars and single units), whereas American first graders tended to use only single unit cubes to represent the same numbers. For example, when shown number 34, Japanese or Chinese children typically represented it with three 10bars and four single units, whereas American children used 34 single units. In contrast, our study showed comparable performance by students from the United States and Taiwan: Both groups primarily produced base-10 representations, indicating a change in American students' performance.

The difference between the findings of the present and earlier studies using the blocks-task is not likely to be due to the socioeconomic characteristics of our participants because the earlier studies also involved students from professional well-educated families. It is possible that a change in American students' performance on the number representation task reflects, in part, the concerted effort to facilitate children's conceptual understanding of numbers and numeric structure that was undertaken by United States educators in the last decades (National Council of Teachers of Mathematics, 2006; National Research Council, 2001). The exact reasons behind this phenomenon, which could be explored in future work, are outside the scope of the present study. What is important is that our participants demonstrated a comparably high ability to represent double-digit numbers as combinations of $10 \mathrm{~s}$ and $1 \mathrm{~s}$ and yet significant differences in the level of use of the decomposition strategy.

Summary: Strategy use in solving addition problems. Our analyses revealed both similarities and differences between Tai- wanese and American first graders in strategy use. The similarities were observed in the patterns of strategy distribution: (a) the use of counting first increased as a function of problem complexity and then decreased as a further increase in complexity rendered this strategy inefficient and error-prone, and (b) the use of decomposition increased from simple to complex problems. The differences were observed in the overall levels of strategy use: Taiwanese students' reliance on counting was lower and their use of decomposition was higher than in their American peers.

The observed similarities in the patterns of strategy use underscore universal principles of mathematical learning and reasoning. In particular, the findings support the idea that strategy choice is governed by a competition between efficiency and accuracy, even when looking at children that differ substantially in performance levels and the frequency of specific strategies. The observed differences, on the other hand, point to a potential role of educational and cultural factors in shaping children's performance within these universal principles.

Although the present study focused on cross-national variability, it is possible that large differences would be found within-countries if children from diverse socioeconomic backgrounds were examined. For the purposes of the present study, the focus on higher SES participants made most sense because it provided a better control of experiential factors at home. Children from well-educated families across countries are likely to receive similarly rich educational experiences at home, including extracurricular opportunities, cognitively stimulating games, and language and literacy exposure (Duncan \& Murnane, 2011). In future research, it would be important to extend this line of inquiry to children from diverse socioeconomic backgrounds. It can be expected that lower income children will use advanced strategies less frequently than their higher income counterparts, but it remains to be seen whether they will reveal a similar pattern of strategy use as a function of problem complexity and a similar relation between strategy and accuracy as found with the current sample. If so, it would provide evidence that even at lower levels of prerequisite skills, children attempt to recruit those skills when faced with more complex mathematical problems.

\section{Cross-National Differences on Complex Arithmetic: Potential Sources and Implications}

On the basis of the analysis of the DD addition task, we expected that the use of decomposition, as well as basic computational fluency, would be strongly associated with accuracy on this task. Further, the extent to which decomposition predicted accuracy could depend on computational fluency. That is, once the child converted a double-digit addition problem into a series of simpler problems, the success in solving these smaller problems depends on how quickly and accurately the child retrieves basic addition facts. Thus, we hypothesized that cross-national differences in the accuracy of children's performance on DD addition may be related to corresponding differences in the use of decomposition strategy and computational fluency, and that these two factors may interact with each other in predicting accuracy.

Our findings confirmed this prediction. The mediation analysis showed that both computational fluency and the use of decomposition strategy mediated the differences between Taiwanese and American students on complex arithmetic. Specifically, Taiwanese students showed a greater ability to retrieve SD number facts and use decomposition than their American peers, and each of these 
factors accounted for a significant portion of cross-national differences in the accuracy of DD addition. Further, both mediators were moderating each other. For example, the positive effect of using a decomposition strategy on accuracy was enhanced by fluencythe students who frequently used this strategy and were highly fluent with basic number facts experienced an additional boost to their performance, compared to those who used decomposition equally frequently but were less fluent.

The cross-national differences in computational fluency and in the use of decomposition strategy observed in the present study could be due to a number of reasons. It has been suggested, for example, that speakers of Chinese and several other Asian languages have advantages in mathematical learning due to certain features of their numeric language (e.g., Miura, 1987). In particular, number words in Chinese are very short, which decreases the working memory demands during computations and may facilitate the process of committing number facts to long-term memory, resulting in greater computational fluency. Furthermore, unlike English, Chinese number words for multidigit numbers contain clear cues about the hierarchical base-10 structure of the numeric system (e.g., the word for 12 is "ten-two"), which offers a general advantage in understanding the numeric structure and a particular advantage in using the base-10 decomposition strategy.

Although the differences between the English and Chinese linguistic systems are impressive, the differences between the corresponding educational contexts are equally striking (Fuson \& Li, 2009; Perry, 2000; Stigler \& Stevenson, 1992). It is possible then that the differences in fluency and in the use of decomposition reflect differences in the curricular approach. An analysis of math lessons in Chinese schools by Perry (2000) offered examples of first-grade teachers commonly highlighting the base-10 decomposition strategy by addressing not just the accuracy of response, but the way in which it was reached. Fuson and Li (2009) reviewed textbooks widely used in China and the United States and found that Chinese textbooks promoted a base-10 decomposition method to a much greater extent than American textbooks.

Although we did not conduct observations of math lessons in the present study, we did examine curricular guidelines and instructional materials as part of a larger research project. We found that, starting with the topics on SD addition, Taiwanese curriculum placed a greater emphasis on the use of base-10 decomposition. Decomposition strategy was also present in instructional materials used with our American participants, but it was typically presented as one of many available strategies (some of which involve manipulatives, others-mental calculation) and there was less practice with decomposition on SD and MD numbers.

Quite likely, curricular differences interact with linguistic factors as well as a host of other cultural factors to produce persistent cross-national differences in mathematics outcomes. Much of the extant cross-national literature has focused on the role of such factors, as educational expectations, parental practices, and teachers' beliefs, in explaining the relation between culture and math performance (e.g., Chen \& Stevenson, 1995; Ng \& Rao, 2010; Wang \& Lin, 2005). Some of these factors, however, are not particularly malleable or proximal to the process of mathematical problem solving. In contrast, the mediators of cross-national differences identified in the present study-computational fluency and frequency of using decomposition-are both malleable and directly involved in the computational process. While acknowl- edging that cultural factors established in prior literature are related to both the predictors and the outcomes of our analysis, we propose that identifying more proximal factors has particular utility for educational practice. Establishing that specific skills taught in elementary school mediate cross-national differences points to concrete intervention targets.

\section{References}

Bailey, D. H., Littlefield, A., \& Geary, D. C. (2012). The codevelopment of skill at and preference for use of retrieval-based processes for solving addition problems: Individual and sex differences from first to sixth grades. Journal of Experimental Child Psychology, 113, 78-92. http:// dx.doi.org/10.1016/j.jecp.2012.04.014

Baroody, A. (2006). Why children have difficulties mastering the basic number combinations and how to help them. Teaching Children Mathematics, 13, 22-31.

Barrouillet, P., \& Lépine, R. (2005). Working memory and children's use of retrieval to solve addition problems. Journal of Experimental Child Psychology, 91, 183-204. http://dx.doi.org/10.1016/j.jecp.2005.03.002

Booth, J. L., \& Siegler, R. S. (2008). Numerical magnitude representations influence arithmetic learning. Child Development, 79, 1016-1031. http://dx.doi.org/10.1111/j.1467-8624.2008.01173.x

Carr, M., \& Alexeev, N. (2011). Fluency, accuracy, and gender predict developmental trajectories of arithmetic strategies. Journal of Educational Psychology, 103, 617-631. http://dx.doi.org/10.1037/a0023864

Case, R. (1993). Theories of learning and theories of development. Educational Psychologist, 28, 219-233. http://dx.doi.org/10.1207/ s15326985ep2803_3

Chen, C., \& Stevenson, H. W. (1995). Motivation and mathematics achievement: A comparative study of Asian-American, CaucasianAmerican, and East Asian high school students. Child Development, 66, 1214-1234. http://dx.doi.org/10.2307/1131808

Cohen, J., Cohen, P., Vest, S. G., \& Aiken, L. S. (2014). Applied multiple regression/correlation analysis for the behavioral sciences. London, England: Routledge.

Cowan, R., Donlan, C., Shepherd, D., Cole-Fletcher, R., Saxton, M., \& Hurry, J. (2011). Basic calculation proficiency and mathematics achievement in elementary school children. Journal of Educational Psychology, 103, 786-803. http://dx.doi.org/10.1037/a0024556

DeStefano, D., \& LeFevre, J. (2004). The role of working memory in mental arithmetic. The European Journal of Cognitive Psychology, 16, 353-386. http://dx.doi.org/10.1080/09541440244000328

Duncan, G. J., \& Murnane, R. J. (Eds.). (2011). Whither opportunity? Rising inequality, schools, and children's life chances. New York, NY: Russell Sage Foundation.

Fischer, K. W. (1980). A theory of cognitive development: The control and construction of hierarchies of skills. Psychological Review, 87, 477-531. http://dx.doi.org/10.1037/0033-295X.87.6.477

Fuson, K. C., \& Kwon, Y. (1992). Korean children's single-digit addition and subtraction: Numbers structured by ten. Journal for Research in Mathematics Education, 23, 148-165. http://dx.doi.org/10.2307/749498

Fuson, K. C., \& Li, Y. (2009). Cross-cultural issues in linguistic, visualquantitative, and written-numeric supports for mathematical thinking. ZDM, 41, 793-808. http://dx.doi.org/10.1007/s11858-009-0183-7

Geary, D. C., Bow-Thomas, C. C., Liu, F., \& Siegler, R. S. (1996). Development of arithmetical competencies in Chinese and American children: Influence of age, language, and schooling. Child Development, 67, 2022-2044. http://dx.doi.org/10.2307/1131607

Geary, D., Fan, L., \& Bow-Thomas, C. (1992). Numerical cognition: Loci of ability differences comparing children from China and the US. Psychological Science, 3, 180-185. http://dx.doi.org/10.1111/j.1467-9280 1992.tb00023.x 
Geary, D. C., Hoard, M. K., Byrd-Craven, J., \& DeSoto, M. C. (2004). Strategy choices in simple and complex addition: Contributions of working memory and counting knowledge for children with mathematical disability. Journal of Experimental Child Psychology, 88, 121-151. http://dx.doi.org/10.1016/j.jecp.2004.03.002

Göbel, S. M., Watson, S. E., Lervåg, A., \& Hulme, C. (2014). Children's arithmetic development: It is number knowledge, not the approximate number sense, that counts. Psychological Science, 25, 789-798. http:// dx.doi.org/10.1177/0956797613516471

Griffin, S. (2009). Learning sequences in the acquisition of mathematical knowledge: Using cognitive developmental theory to inform curriculum design for pre-K-6 mathematics education. Mind, Brain, and Education, 3, 96-107. http://dx.doi.org/10.1111/j.1751-228X.2009.01060.x

Hayes, A. F. (2013). Introduction to mediation, moderation, and conditional process analysis. New York, NY: Guilford Press.

Hayes, A. F., Preacher, K. J., \& Myers, T. A. (2011). Mediation and the estimation of indirect effects in political communication research. In E. P. Bucy \& R. Lance Holbert (Eds.), Sourcebook for political communication research: Methods, measures, and analytical techniques (pp. 434-465). New York, NY: Routledge.

Imbo, I., \& Vandierendonck, A. (2007). The development of strategy use in elementary school children: Working memory and individual differences. Journal of Experimental Child Psychology, 96, 284-309. http:// dx.doi.org/10.1016/j.jecp.2006.09.001

Jordan, N. C., Hanich, L. B., \& Kaplan, D. (2003). Arithmetic fact mastery in young children: A longitudinal investigation. Journal of Experimental Child Psychology, 85, 103-119. http://dx.doi.org/10.1016/S00220965(03)00032-8

Jordan, N., Kaplan, D., Locuniak, M., \& Ramineni, C. (2007). Predicting first-grade math achievement from developmental number sense trajectories. Learning Disabilities Research \& Practice, 22, 36-46. http://dx .doi.org/10.1111/j.1540-5826.2007.00229.x

Laski, E., Ermakova, A., \& Vasilyeva, M. (2014). Early use of decomposition strategy for addition and its relation to base-10 knowledge. Journal of Applied Developmental Psychology, 35, 444-454. http://dx.doi .org/10.1016/j.appdev.2014.07.002

Leung, F. K. (2001). In search of an East Asian identity in mathematics education. Educational Studies in Mathematics, 47, 35-51. http://dx.doi .org/10.1023/A:1017936429620

Lindberg, S., Linkersdörfer, J., Lehmann, M., Hasselhorn, M., \& Lonnemann, J. (2013). Individual differences in children's early strategy behavior in arithmetic tasks. Journal of Educational and Developmental Psychology, 3, 192-200. http://dx.doi.org/10.5539/jedp.v3n1p192

MacKinnon, D. P. (2008). Statistical mediation analysis [Introduction]. Mahwah, NJ: Lawrence Erlbaum.

Miura, I. T. (1987). Mathematics achievement as a function of language. Journal of Educational Psychology, 79, 79-82. http://dx.doi.org/10 $.1037 / 0022-0663.79 .1 .79$

Miura, I. T., \& Okamoto, Y. (1989). Comparisons of US and Japanese first graders' cognitive representation of number and understanding of place value. Journal of Educational Psychology, 81, 109-114. http://dx.doi .org/10.1037/0022-0663.81.1.109

Miura, I., Okamoto, Y., Kim, C., Steere, M., \& Fayol, M. (1993). First graders' cognitive representation of number and understanding of place value: Cross-national comparisons-France, Japan, Korea, Sweden, and the United States. Journal of Educational Psychology, 85, 24-30. http:// dx.doi.org/10.1037/0022-0663.85.1.24

Mullis, I. V. S., Martin, M. O., Foy, P., \& Arora, A. (2012). TIMSS 2011 international results in mathematics. Chestnut Hill, MA: TIMSS \& PIRLS International Study Center.
National Council of Teachers of Mathematics. (2006). Curriculum focal points for prekindergarten through grade 8 mathematics. Reston, VA: National Council of Teachers of Mathematics.

National Mathematics Advisory Panel. (2008). Foundations for success: The final report of the National Mathematics Advisory Panel. Washington, DC: U. S. Department of Education.

National Research Council. (2001). J. Kilpatrick, J. Swafford, and B. Findell (Eds.). Adding it up: Helping children learn mathematics. Washington, DC: National Academy Press.

Ng, S. S., \& Rao, N. (2010). Chinese number words, culture, and mathematics learning. Review of Educational Research, 80, 180-206. http:// dx.doi.org/10.3102/0034654310364764

Perry, M. (2000). Explanations of mathematical concepts in Japanese, Chinese, and US first- and fifth-grade classrooms. Cognition and Instruction, 18, 181-207. http://dx.doi.org/10.1207/S153269 0XCI1802_02

Preacher, K. J., \& Hayes, A. F. (2004). SPSS and SAS procedures for estimating indirect effects in simple mediation models. Behavior Research Methods, Instruments, \& Computers, 36, 717-731. http://dx.doi .org/10.3758/BF03206553

Preacher, K. J., \& Hayes, A. F. (2008). Contemporary approaches to assessing mediation in communication research. In A. F. Hayes, M. D. Slater, \& L. B. Snyder (Eds.), The Sage sourcebook of advanced data analysis methods for communication research (pp. 13-54). Thousand Oaks, CA: Sage. http://dx.doi.org/10.4135/9781452272054.n2

Rittle-Johnson, B., \& Siegler, R. S. (1999). Learning to spell: Variability, choice, and change in children's strategy use. Child Development, 70 332-348. http://dx.doi.org/10.1111/1467-8624.00025

Rosenthal, R., \& Rosnow, R. L. (1991). Essentials of behavioral research: Methods and data analysis. Boston, MA: McGraw-Hill.

Salkind, N. J. (2010). Encyclopedia of research design. Thousand Oaks, CA: Sage. http://dx.doi.org/10.4135/9781412961288

Saxton, M., \& Towse, J. N. (1998). Linguistic relativity: The case of place value in multi-digit numbers. Journal of Experimental Child Psychology, 69, 66-79. http://dx.doi.org/10.1006/jecp.1998.2437

Shrager, J., \& Siegler, R. (1998). A model of children's strategy choices and strategy discovery. Psychological Science, 9, 405-410. http://dx.do .org/10.1111/1467-9280.00076

Siegler, R. S. (1987). The perils of averaging data over strategies: An example from children's addition. Journal of Experimental Psychology: General, 116, 250-264. http://dx.doi.org/10.1037/0096-3445.116.3.250

Siegler, R. S. (1996). Emerging minds. The process of change in children's thinking. New York, NY: Oxford University Press.

Stevenson, H. W., Chen, C., \& Lee, S. Y. (1993). Mathematics achievement of Chinese, Japanese, and American children: Ten years later Science, 259, 53-58. http://dx.doi.org/10.1126/science.8418494

Stigler, J. W., \& Stevenson, H. W. (1992). The learning gap: Why our schools are failing and what we can learn from Japanese and Chinese education. New York, NY: Summit Books.

Wang, J., \& Lin, E. (2005). Comparative studies on U.S. and Chinese mathematics learning and the implications for standards-based mathematics teaching reform. Educational Researcher, 34, 3-13. http://dx.doi .org/10.3102/0013189X034005003

Whang, P. A., \& Hancock, G. R. (1997). Modeling the mathematics achievement of Asian-American elementary students. Learning and Individual Differences, 9, 63-88. http://dx.doi.org/10.1016/S10416080(97)90020-0

Received December 10, 2014

Revision received June 15, 2015 Accepted July 16, 2015 